\title{
Melting a granular glass by cooling
}

\author{
Jan Plagge and Claus Heussinger \\ Institute for Theoretical Physics, Georg-August University of Göttingen, Friedrich-Hund Platz 1, 37077 Göttingen
}

(Dated: November 12, 2018)

\begin{abstract}
Driven granular systems readily form glassy phases at high particle volume fractions and low driving amplitudes. We use computer simulations of a driven granular glass to evidence a re-entrance melting transition into a fluid state, which, contrary to intuition, occurs by reducing the amplitude of the driving. This transition is accompanied by anomalous particle dynamics and super-diffusive behavior on intermediate time-scales. We highlight the special role played by frictional interactions, which help particles to escape their glassy cages. Such an effect is in striking contrast to what friction is expected to do: reduce particle mobility by making them stick.
\end{abstract}

PACS numbers:

Friction implies stability. A solid block only remains static on an inclined plane because there is friction with the underlying surface. Similarly, a heap made of granular particles, in general, will have a higher angle of stability with increased friction coefficient 1]. Inside the heap, the particle volume fraction will generally be lower and the number of inter-particle contacts smaller, while the heap nevertheless remains stable [2]. A similar stabilization occurs when the grains are driven into a fluid state. Under shear, the jamming transition from a freely flowing state to a yield-stress fluid occurs at lower volume fractions as compared to the frictionless case [3-5].

Here, we present simulations of a driven granular system were friction acts opposite to what is expected from these simple examples. We show how friction can lead to anomalous particle dynamics that very efficiently fluidize the system. As a result the system undergoes a re-entrance melting transition from a glassy to a fluid state by lowering the amplitude of driving.

Experimentally, a variety of driving mechanisms have been proposed to characterize the dynamical properties of dense granular systems. Among those are shaking [6, 7] fluid- or air-flow [8-10], cyclic shear [11, 12] or temperature oscillations [13, 14]. We describe a two-dimensional system, similar to the setup used in Refs. [6, 9, 10, 15]. At high densities and low enough driving amplitude these systems readily form glassy states, where structural relaxation is completely suppressed [16]. Interestingly, Ref. 15] also reports anomalous particle dynamics deep in the glassy phase, and suspects friction to play a central role in this process. Unfortunately, it is rather difficult experimentally to quantitatively characterize or tune the frictional interactions between the particles [17] or between particles and container walls. The connection between friction and particle dynamics is therefore unclear. Our simulations have the goal to elucidate such a connection.

Model - We consider a monolayer of $N=2500$ disks. One half of the particles ("small") have radius $R_{s}=0.5 d$ and mass $m_{s}=\rho(4 \pi / 3) R_{s}^{3}$, the other half ("large") have radius $R_{l}=0.7 d$ and mass $m_{l}=\rho(4 \pi / 3) R_{l}^{3}$. The parti- cle area fraction is defined as $\phi=\sum_{i=1}^{N} \pi R_{i}^{2} / L^{2}$, where $L$ is the size of the simulation box. Unless otherwise stated we fix $\phi=0.825$, which is only slightly below the random close packing value of $\phi_{c}=0.84$. Periodic boundary conditions are used in both directions.

Particles interact via a standard spring-dashpot interaction (e.g. [2, 4, 5]). In short, two particles $i, j$ interact when they are in contact, i.e. when their mutual distance $r$ is smaller than the sum of their radii $R_{i}+R_{j}$. The interaction force has both a normal component $F_{n}=k_{n}\left(r-\left(R_{i}+R_{j}\right)\right)$ and a tangential component $F_{t}=k_{t} \delta_{t}$, where $k_{n}$ and $k_{t}$ are the spring constants and $\delta_{t}$ is the tangential (shear) displacement since the formation of the contact. The tangential spring mimics sticking of the two particles due to dry friction. The frictional forces are limited by the Coulomb condition $F_{t} \leq \mu F_{n}$, which is implemented by rescaling the tangential displacement $\delta_{t} \rightarrow \mu F_{n} / k_{t}$ whenever necessary.

To mimic the presence of an external container, we place the particles on a flat surface ( $x y$-plane) which acts like a frictional particle with infinite radius. The normal component of the interaction is set by gravity, $F_{n}^{(s)}=m_{i} g$, which pushes the particles into the surface. Therefore, and because we do not allow particles to move away from the surface ( $z$-direction), particles are in permanent contact with the surface. The tangential displacement $\delta_{t}$ then corresponds simply to the in-plane displacement of the particle, properly rescaled when the Coulomb condition is violated, $\delta_{t} \leq \mu_{s} m_{i} g / k_{t}$, where $\mu_{s}$ is the surface friction coefficient.

Particles are driven with an oscillating force $F(t)=$ $A \sin (\omega t)$ that acts (in the plane of the surface) along the $\mathrm{y}$-direction. Even though this driving is uni-directional, we find that due to the dense packing the system remains roughly isotropic. With this kind of driving force, an isolated particle on a frictionless surface oscillates at an amplitude $y_{0}=A / m \omega^{2}$. In a dense assembly this leads to local frustration as particles with smaller masses tend to move faster. Rearrangements result which, at the high densities under consideration, may or may not be able to lead to structural relaxation. It is this glassy dynamics 
that we are interested in, with the driving amplitude $A$ playing the role of thermal temperature. Note, that this driving mechanism injects energy directly into the bulk. System-size is therefore not an issue and the system is spatially homogeneous.

As units we choose particle mass density $\rho$, particle diameter $d$ and the period of the driving, $T=2 \pi / \omega$. With these definitions we perform molecular dynamics simulations using LAMMPS [18] with parameters $k_{n}=$ $1000, k_{t}=2 k_{n} / 7$ and a time-step of $\Delta t=0.001$.

The simplest quantity which is measured from particle displacements is the mean-squared displacement (MSD),

$$
\Delta^{2}(t)=\left\langle\frac{1}{N} \sum_{i=1}^{N}\left[x_{i}\left(t_{0}+t\right)-x_{i}\left(t_{0}\right)\right]^{2}\right\rangle
$$

where $\Delta x_{i}\left(t_{0}, t\right)=x_{i}\left(t+t_{0}\right)-x_{i}\left(t_{0}\right)$ is the displacement of particle $i$ in the time interval $\left[t_{0}, t_{0}+t\right]$ in the direction transverse to the driving. Snapshots of the system are taken after every full force cycle. Time is therefore restricted to $t \equiv t_{n}=n T$ and $n$ integer. With this definition the MSD is zero when particle motion during cycles is periodic.

Results - Let us first consider the case where there is no inter-particle friction $(\mu=0)$ and only particle-surface friction $\left(\mu_{s}=1\right)$. In Fig. [1 we display the evolution of the MSD for various driving amplitudes $A$.

At relatively high amplitudes $(A \geq 3)$ we observe typical signs of glassy dynamics; an extended plateau in the MSD, caging of particles and hopping events (see sample trajectory in Fig. 17 inset). As expected the MSD decreases with decreasing driving amplitude. Surprisingly, this trend does not persist when the amplitude is further reduced. Instead, for lower amplitudes we observe a greatly enhanced MSD and a total dissapearance of the plateau. Thus, the system is fluidized, very unintuitively, by reducing the amplitude of agitation (also see suppl. movies). This re-entrance melting transition is our main result.

The fluidization is particularly evident in the long-time diffusivity $D:=\lim _{t \rightarrow \infty} \Delta^{2}(t) / t$ (see Fig 2), which has a minimum in the glassy regime 21] but then strongly increases up to a sharp maximum at $A^{\star} \approx 1$. At the same time, the "granular temperature" $T$, defined as the average kinetic energy in the direction transverse to the drive, is completely normal and monotonously decreases with decreasing $A$. This parallels the behavior of the MSD at short times. Anomalous dynamics only develops at intermediate time-scales. Associated with the maximum of $D\left(A^{\star}\right)$, we observe an intermediate super-diffusive regime that spans one to two orders of magnitude in time.

Discussion - The external driving injects momentum into the system. This momentum is subsequently randomized by inter-particle collisions, and dissipated by surface friction. The onset of re-entrance melting corresponds to the situation that dissipation of momentum
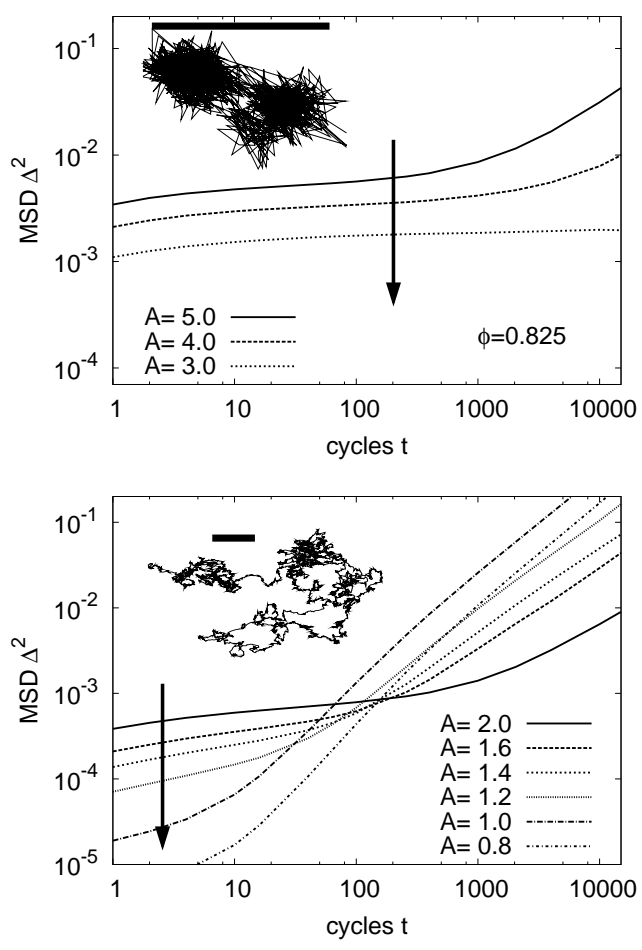

FIG. 1: Mean-square displacement $\Delta^{2}(t)$ for different driving amplitudes $A$ (arrows indicate decreasing amplitude). At short times the plateau decreases with decreasing amplitude. By way of contrast, at long times the MSD is nonmonotonic with $A$ and has a maximum at $A^{\star} \approx 1$. On intermediate timescales superdiffusive particle motion develops when $A \approx A^{\star}$. Inset: typical trajectories in glassy regime (top) and in the re-entrance fluid (bottom). Scale-bar is of length $R_{s}$.

competes with the randomization due to collisions. This can most easily be seen in a system, where only the small particles are driven. The large particles then only move because they are kicked around by the mobilized small particles. We can show (see suppl. material) that in the re-entrance fluid phase kicks only temporarily mobilize the large particles. They then undergo some small slip displacement and quickly come to rest before the next collision occurs. Thus, all the momentum from the collision is immediately lost to the surface. By way of contrast, in the glassy phase this momentum is first redistributed to other particles before it is dissipated away.

Similar effects occur when both particles are driven. The value of $A^{\star}=1$ is below the Coulomb threshold for the large particles, which in our units is at a force $A_{l}=1.43$. This means that large particles can only be mobilized by additional kicks from mobile small particles, which have a smaller Coulomb threshold of $A_{s}=0.52$. Accordingly, the MSD of large particles is suppressed on short times (Fig 3 ) but otherwise displays the same superdiffusive behavior on intermediate time-scales.

Superdiffusion then naturally emerges, when 


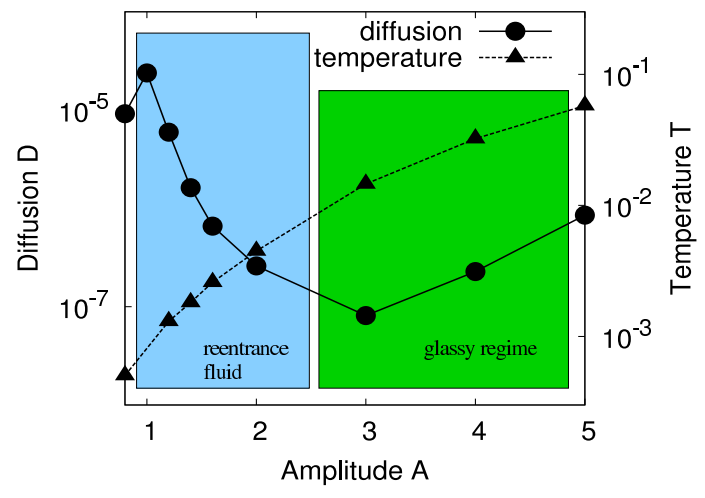

FIG. 2: The effective diffusivity $D$ is nonmonotonous with a minimum in the glassy regime and a maximum at $A^{\star} \approx 1$. The temperature $T$ is monotonously decreasing with decreasing amplitude.
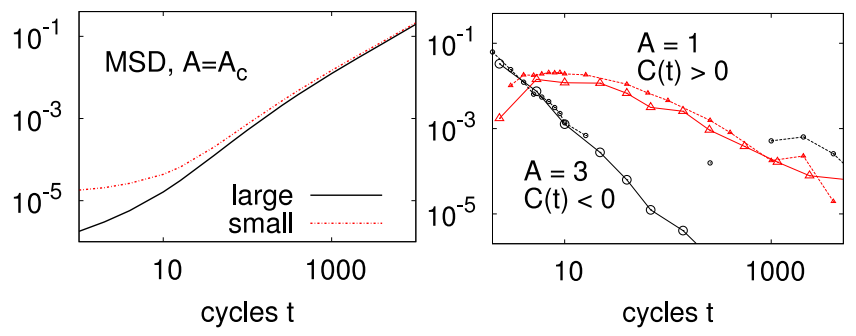

FIG. 3: (left) MSD of large particles is similar to that of small particles, except at short times, where large particles move much less. (right) Normalized autocorrelation function $C(\tau) / C(0)$ of single-cycle displacements. Negative correlations in glassy regime $(A=3$, plotted is $-C(\tau))$ compare with positive correlations in re-entrance fluid $(A=1)$. Data taken by direct evaluation of the correlations (small symbols) and by differentiation of MSD, via $C(\tau)=\partial^{2} \Delta^{2}(\tau) / \partial \tau^{2}$ (large symbols).

there is positive temporal correlations in the kicks. Fig. 3 displays the auto-correlation function $C(t)=\left\langle\Delta x\left(t_{0}, 1\right) \Delta x\left(t_{0}+t, 1\right)\right\rangle$ of large-particle displacements, $\Delta x(t, 1)$, during a single cycle. Clearly, a pronounced positive correlation is visible at intermediate times corresponding to the superdiffusive regime. The same positive correlations are visible in the trajectory displayed in Fig.1b (inset) where they lead to long stretches of quasi-directed motion. By way of contrast, the correlation function is negative in the glassy regime, indicating anti-correlations in the kicks.

To understand the origin of these correlations, we have to analyze in more detail the dynamics of the small particles. With the large particles sticking to the surface, the small particles explore their local free volume on short times within an effectively frozen environment. This situation is depicted in Fig 4 , where a small test particle (black dots) is confined to a typical cage-like surrounding, which is taken to consist of large particles that are
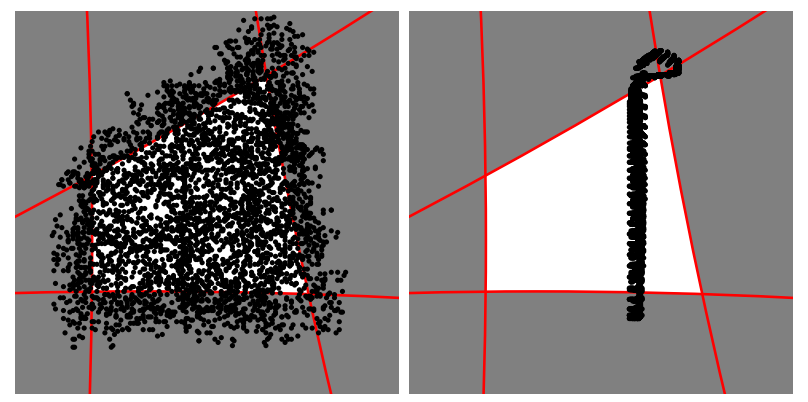

FIG. 4: Short-time $(t=30)$ motion of a small test particle (black dots) as driven by the periodic force without (left) and with surface friction (right). The test particle is confined by a set of large particles, which are frozen in space. The "excluded" space (grey area) is formed from the area covered by "effective" particles of radius $R_{s}+R_{l}$. For the high densities under consideration, the available space for particle motion (white area) is much smaller than the particle diameter. The boundaries therefore appear on this scale only with a small curvature (red lines). As the particles are not infinitely stiff, some overlap during collisions (penetration into the grey area) is allowed.

frozen in space (grey area and lines). Driven by the external force the test particle will move around and explore the available free volume (white area).

If we switch off the frictional interactions of the test particle with the underlying surface (Fig 4 a), the motion is quickly randomized by the collisions with the cage. This builds up a pore pressure, which can act as a restoring force when, on longer time-scales, the large particles (cage wall) start to move. The consequences are anticorrelation (negative $C(\tau)$ ), particle localization and caging.

With friction turned on (Fig 4 ), the trajectory is completely different and the test particle explores only a small part of the available volume. Any additional momentum from an inter-particle collision is quickly dissipated and the particles stick to the surface for as long as the force needs to switch sign and push it back. This allows to synchronize with the force and avoids the chaotic type of motion characteristic of the glassy regime. The synchronization is apparent in the phase of the oscillations of the particle. Without friction the particle coordinate is out-of phase with the forcing, just like a driven and undamped oscillator. With friction the particle velocity is in phase with the forcing, like an overdamped oscillator (see suppl. material).

On intermediate time-scales the large particles move and the cage evolves. If the kicks are sufficiently weak (small $A$ ) the cage will only change very little from one cycle to the next and the periodic trajectories of the small particles slowly evolve with the surrounding structure. On this intermediate time-scale the kicks are correlated and lead to superdiffusive motion of the confining large particles (and as a consequence also of the confined small 


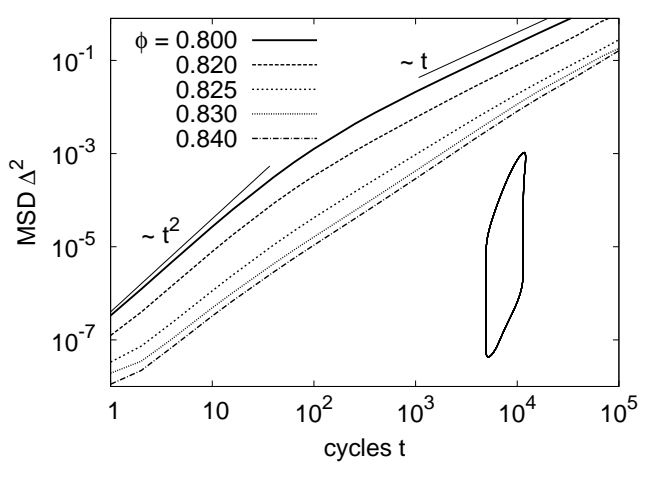

FIG. 5: MSD with interparticle friction and strong damping $\zeta=50$ chosen such that velocities are damped on time-scales shorter than the driving period. The driving force $(A=50)$ is only applied to small particles. If all particles were driven, no rearrangements would occur (overdamped limit). The MSD decreases upon increasing the volume-fraction towards the random-close packing limit. Inset: example of a periodic particle trajectory when friction is switched off completely. The amplitude of the motion corresponds to roughly $5 \%$ of the particle diameter.

particles). With increasing driving amplitude the kicks get stronger and stronger, and the positive correlation is reduced. Finally, at high enough amplitudes the large particles are fully mobilized and cannot dissipate their momentum before the next kick occurs. This completely randomizes particle motion and corresponds to the transition into the glassy regime.

Inter-particle friction - The above discussion of the origin of anomalous diffusion shows that surface friction essentially acts as a strong dissipation mechanism. However, if we substitute surface friction with a strong linear damping force, $\vec{F}_{\text {damp }}=-\zeta \vec{v}_{i}$, and in the absence of inter-particle friction $(\mu=0)$, no anomalous dynamics occurs. In fact, the particles move on strictly periodic trajectories (Fig. 15 inset) and the MSD is identical zero. Such a behavior parallels the caging dynamics seen in the glassy regime, with the effective cage shrinking to a point. This suggests that the nonlinear nature of friction is also essential for the anomalous dynamics. Indeed, if we switch on inter-particle friction $(\mu=1)$, particles readily diffuse around and the MSD displays again a super-diffusive (or even ballistic) regime at short and intermediate time-scales (Fig. 河). The second role of friction, next to dissipation, is thus to induce small perturbations during particle collisions, such that the periodic trajectories are slightly, but irreversibly modified. This leads to a slow but steady evolution of the local structure, which is visible in the MSD as ballistic regime.

Conclusion - We have shown that, quite unintuitivly, frictional interactions can speed up particle motion and lead to superdiffusive dynamics. This contrasts with what friction is expected to do: slow down particle mo- tion by making them stick. We have rationalized this nontrivial behavior by considering friction as a weak irreversible perturbation to genuinely periodic trajectories. This fluidization competes with the localization due to randomized particle motion. As a result we encounter a re-entrance melting transition from a granular glass (localized) to a fluid state upon lowering the temperature.

These results may be important for experiments in several ways. First, note that the role of frictional interactions with the surface is quite subtle. Fluidization only happens on relatively long time-scales, while on short times, particle motion is completely normal. There, friction only leads to a suppression of particle motion because of the Coulomb threshold. Such effects would make it hard to evaluate the relevance of friction in experiments, whenever interactions with an external container are to be avoided.

In the experiments of Ref. [15] superdiffusive particle motion was indeed observed, however only at volume fractions around the critical close packing limit. In contrast, we find anomalous dynamics for a range of densities (Fig. 5). The high stiffness of the brass particles in the experiment makes a key difference with the simulation. We speculate that only at $\phi_{c}$ this high stiffness provides a sufficiently tight packing, such that frictional effects can compete with the randomization due to the driving. The role of friction would then be to provide the nonlinear ingredient that can "rectify" the motion. Our analysis suggests that important additional insight can be obtained by changing the driving amplitude. First results 19] indeed suggest that the anomalous dynamics is enhanced for lower driving amplitudes.

Finally, the transition between reversible and irreversible particle motion is a nice example of a transition into an absorbing state, as described in the experiments of Pine et al. 20]. Interestingly, in our case the particles continue to interact in the absorbing state. This can, for example, be seen from the periodic trajectories of individual particles, which are complex loops and not just straight lines (inset of Fig 5 ). It remains to be seen if a volume fraction can be identified, which plays the role of the critical point of this non-equilibrium transition. We leave this question for future work.

We acknowledge support by the Deutsche Forschungsgemeinschaft, Emmy Noether program: He 6322/1-1.

[1] R. M. Nedderman, Statics and kinematics of granular materials, Cambridge University Press (1992).

[2] L. E. Silbert, D. Ertas, G. S. Grest, T. C. Halsey, and D. Levine, Phys. Rev. E 65, 031304 (2002),

[3] D. Bi, J. Zhang, B. Chakraborty, and R. P. Behringer, Nature 480, 355 (2011).

[4] M. Otsuki and H. Hayakawa, Phys. Rev. E 83, 051301 (2011), 
[5] M. P. Ciamarra, R. Pastore, M. Nicodemi, and A. Coniglio, Phys. Rev. E 84, 041308 (2011),

[6] P. M. Reis and T. Mullin, Phys. Rev. Lett. 89, 244301 (2002)

[7] C. M. Pooley and J. M. Yeomans, Phys. Rev. Lett. 93, 118001 (2004)

[8] M. Schröter, D. I. Goldman, and H. L. Swinney, Phys. Rev. E 71, 030301 (2005).

[9] A. S. Keys, A. R. Abate, S. C. Glotzer, and D. J. Durian, Nat. Phys. 3, 260 (2007).

[10] F. Lechenault and K. E. Daniels, Soft Matter 6, 3074 (2010),

[11] O. Pouliquen, M. Belzons, and M. Nicolas, Phys. Rev. Lett. 91, 014301 (2003).

[12] O. Dauchot, G. Marty, and G. Biroli, Phys. Rev. Lett. 95, 265701 (2005).

[13] K. Chen, J. Cole, C. Conger, J. Draskovic, M. Lohr, K. Klein, T. Scheidemantel, and P. Schiffer, Nature 442,
257 (2006).

[14] T. Divoux, H. Gayvallet, and J.-C. Géminard, Phys. Rev. Lett. 101, 148303 (2008).

[15] F. Lechenault, O. Dauchot, G. Biroli, and J.-P. Bouchaud, Europhys. Lett. 83, 46002 (2008).

[16] W. T. Kranz, M. Sperl, and A. Zippelius, Phys. Rev. Lett. 104, 225701 (2010),

[17] S. Utermann, P. Aurin, M. Benderoth, C. Fischer, and M. Schröter, Phys. Rev. E 84, 031306 (2011),

[18] http://lammps.sandia.gov/index.html.

[19] C. Coulais, R. P. Behringer and O. Dauchot, arXiv:1202.5687v2 (2012).

[20] D. J. Pine, J. P. Gollub, J. F. Brady, and A. M. Leshansky, Nature 438, 997 (2005).

[21] In this regime we cannot reach the long-time diffusive regime and $D$ is only an upper bound for the real diffusivity. 